ARTICLE

https://doi.org/10.1038/s41467-019-14195-x

\title{
Cerebrospinal fluid tracer efflux to parasagittal dura in humans
}

\author{
Geir Ringstad (D) ${ }^{1} \&$ Per Kristian Eide (iD ${ }^{2,3 *}$
}

The mechanisms behind molecular transport from cerebrospinal fluid to dural lymphatic vessels remain unknown. This study utilized magnetic resonance imaging along with cerebrospinal fluid tracer to visualize clearance pathways to human dural lymphatics in vivo. In 18 subjects with suspicion of various types of cerebrospinal fluid disorders, 3D T2-Fluid Attenuated Inversion Recovery, T1-black-blood, and T1 gradient echo acquisitions were obtained prior to intrathecal administration of the contrast agent gadobutrol $(0.5 \mathrm{ml}, 1 \mathrm{mmol} /$ $\mathrm{ml}$ ), serving as a cerebrospinal fluid tracer. Propagation of tracer was followed with $\mathrm{T} 1$ sequences at 3, 6, 24 and $48 \mathrm{~h}$ after the injection. The tracer escaped from cerebrospinal fluid into parasagittal dura along the superior sagittal sinus at areas nearby entry of cortical cerebral veins. The findings demonstrate that trans-arachnoid molecular passage does occur and suggest that parasagittal dura may serve as a bridging link between human brain and dural lymphatic vessels.

\footnotetext{
${ }^{1}$ Department of Radiology and Nuclear Medicine, Oslo University Hospital - Rikshospitalet, Oslo, Norway. ${ }^{2}$ Department of Neurosurgery, Oslo University Hospital-Rikshospitalet, Oslo, Norway. ${ }^{3}$ Institute of Clinical Medicine, Faculty of Medicine, University of Oslo, Oslo, Norway. ${ }^{*}$ email: p.k.eide@medisin.uio.no
} 
T he human subarachnoid space is shown to be continuous with the paravascular compartment of all brain regions ${ }^{1}$, enabling for cerebrospinal fluid (CSF) to serve as carrier of solutes from brain metabolism into subarachnoid space. Clearance from CSF to blood was for long considered to be dual; one directly to venous blood through arachnoid granulations, and one via lymphatic channels along exiting nerves at the skull base and from the spine ${ }^{2}$. Lymphatic CSF drainage, and the interplay between the lymphatic- and central nervous system (CNS), gained renewed interest when true lymphatic vessels were shown to reside within the dura mater ${ }^{3,4}$. Subsequently, impairment of these meningeal lymphatic pathways has been shown to slow paravascular efflux of amyloid- $\beta$ from interstitial brain fluid and may possibly represent an aggravating factor in Alzheimer's disease and age-associated cognitive decline ${ }^{5}$. Neuromodulators from meningeal lymphatics can even possibly modulate brain tissue by their direct release into $\mathrm{CSF}^{6}$. A recent animal study was, however, unable to reproduce passage of CSF tracer into dural lymphatic vessels ${ }^{7}$ and was thus in line with previous observations indicating that the arachnoid constitutes an impermeable barrier layer between the CSF and dura mater ${ }^{8,9}$.

Our aim was to explore how these diverging findings of CSF tracer efflux from animal studies translate into a human cohort. We carried out multi-phase magnetic resonance imaging (MRI) for up to $48 \mathrm{~h}$ in order to follow the movements of the MRI contrast agent gadobutrol, serving as a CSF tracer. Gadobutrol is a non-ionic, highly hydrophilic molecule of relatively small size $(604 \mathrm{Da})$, prevented by the intact brain-blood-barrier from leakage into CNS blood vessels when administered intrathecally ${ }^{10}$. The substance is therefore suitable for tracing molecular efflux from the cranial compartment along extra-vascular pathways.

\section{Results}

Patients. The study included 18 patients who were under clinical work-up for possible CSF disorders of various etiologies (Supplementary Table 1).

CSF tracer movement in CSF spaces. After intrathecal injection of the CSF tracer, its movement over time via foramen magnum and underneath the base of the brain toward the upper medial cerebral convexities is illustrated in Supplementary Movie 1.

Parasagittal dura. Tissue within the parasagittal dura having features as specified above (intermediate or high signal at T2FLAIR (not free CSF) and at T1-BB (not blood)) was visually identified almost exclusively along the middle to posterior segment of the superior sagittal sinus (Fig. 1; Supplementary Movie 2), corresponding well with the levels where cortical veins enter the sinus.

Even though there were some variations with regard to prominence, tissue in parasagittal dura with features as here defined was depicted in 18/18 study patients and had the typical appearance of longitudinally interspersed areas of up to several millimeters' transversal width (Fig. 2a, b). After intrathecal injection of CSF tracer, we found enrichment of parasagittal dura at 6,24 and $48 \mathrm{~h}$ and with peak at $24 \mathrm{~h}$ (Fig. $2 \mathrm{c}-\mathrm{h}$, Tables 1,2). At group level, there was a highly significant increase in normalized T1 signal units within the parasagittal dura, though more pronounced in nearby CSF (Fig. 2i).

As expected, CSF tracer also enhanced in macroscopically visible arachnoid granulations, bulging into the sagittal sinus in scattered locations (Fig. 3). As can be noted, the signal change differed somewhat within the CSF of the subarachnoid space, arachnoid granulations and parasagittal dura, suggesting some level of molecular motion restriction between these structures. Supplementary Movie 3 presents an animation of an arachnoid granulation bulging from parasagittal dura in the superior sagittal sinus.

The tracer enrichment in parasagittal dura was highly dependent on presence of tracer in adjacent CSF, which was shown by their strong positive correlation at all time points $(3,6$, 24 and 48 h) (Fig. 4). At late scans (i.e. 24 and 48 h, respectively), the remnants of tracer in CSF typically accumulated at the high cerebral convexities and in regions adjacent to parasagittal dura (Fig. 1). Tubular structures with any resemblance to dural lymphatic vessels as previously reported could not be discriminated from other enhancing tissue within parasagittal dura, and may be related to the image resolution of our MRI scans being restricted to $1 \times 1 \times 1 \mathrm{~mm}$ voxel size.

For the visual assessment of tracer enhancement on T1-BB below selected cranial nerve outlets at the skull base, this was detected below the hypoglossal canal in 17/18, the jugular foramen in $17 / 18$, the cribriform plate in $2 / 18$, and the foramen ovale in 0/18 individuals (Fig. 5).

\section{Discussion}

This study demonstrates for the first time direct efflux of a CSF tracer to parasagittal dura in humans. The findings render for the parasagittal dura to represent a bridging link for CSF-mediated exchange of molecules between brain tissue and dural lymphatic vessels.

The extent of enhancing tissue within parasagittal dura does not resemble the previously reported appearance of true dural lymphatic vessels, which are tubular-shaped structures of submillimeter cross-sectional diameter near the venous sinus wall ${ }^{3,4,11}$, and also far from the classical MRI appearance of arachnoid granulations bulging into venous sinuses ${ }^{12}$. Our findings compare better with previous anatomical reports demonstrating that parasagittal dura along the middle one-third of the superior sagittal sinus harbors a dense carpet of arachnoid granulations medially, being in close association with an extensive network of intradural channels from 0.02 to $2.0 \mathrm{~mm}$ in diameter, coursing and coalescing into lateral lacunae from laterally toward the superior sagittal sinus ${ }^{13,14}$ (illustrated in Fig. 6). The endothelium of intradural channels was proposed to possibly be the final barrier for the absorption of CSF to venous blood ${ }^{15}$, but passage of CSF over this cell layer has never since been confirmed in vivo. The in vivo-based observations from the current MRI study show that parasagittal dura does not drain CSF only, but also molecules, with high level of dependence on their presence in the adjacent subarachnoid compartment. In light of more recent discoveries showing evidence of true dural lymphatic vessels in the superior sagittal sinus wall 3,4 , and the fact that lymphatic vessels as well as blood vessels within the dura lack tight junctions ${ }^{16}$, it seems plausible that molecules within parasagittal dura structures may well reach adjacent lymphatic vessels, and perhaps also vice versa. The parasagittal dura may thus represent a bridging link for CSF-mediated exchange of molecules between brain tissue and dural lymphatic vessels.

The region with tracer enhancement, as presented here, stretches laterally to a larger extent than what can be anticipated from the previous anatomical descriptions of intradural human arachnoid granulations ${ }^{14,15}$, which should be highly suggestive for tracer escape to dura outside arachnoid granulations and into the network of intradural channels. To this end, we note that any CSF or molecular escape to arachnoid granulations whatsoever has lately been questioned $^{7}$, and explained by commonly accepted assumption of the impermeable arachnoid-barrier. 


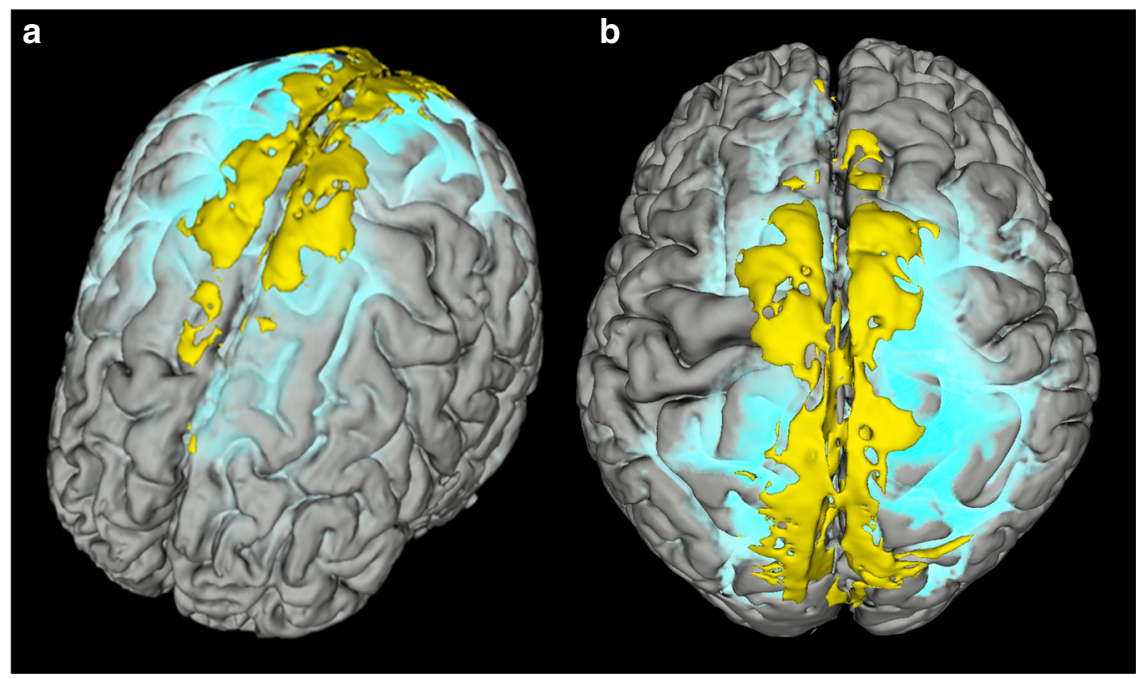

Fig. 1 3D representation of parasagittal dura as defined from T2-FLAIR. The brain is shown in oblique coronal view (a) and viewed from above (b). Parasagittal dura (dark yellow) as defined from T2-FLAIR and co-registered with rough segmentation of the brain (gray) and tracer enhancement in CSF (light blue) from T1 GRE at $48 \mathrm{~h}$ after intrathecal administration. The CSF tracer propagated toward the upper brain convexities and parasagittal dura at late time points (at $24 \mathrm{~h}$ and particularly at $48 \mathrm{~h}$ ). A video-animation presenting the full longitudinal extension of parasagittal dura is presented in Supplementary Movie 2. Images: Tomas Sakinis, MD.
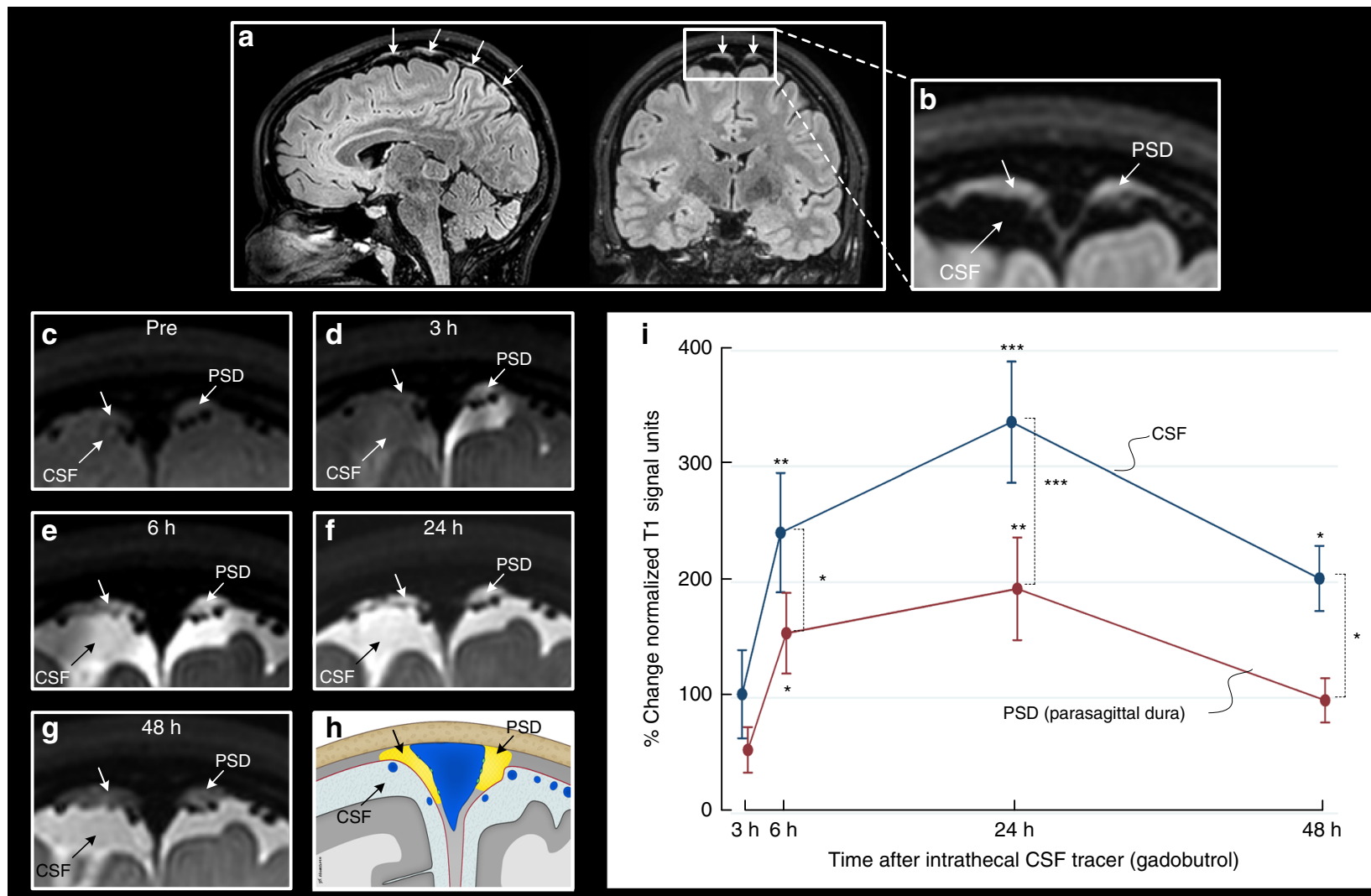

Fig. 2 Cerebrospinal fluid (CSF) tracer efflux to parasagittal dura in humans. a Sagittal and coronal T2-FLAIR MR images, respectively, show the typical longitudinal and lateral extension of parasagittal dura (PSD) with high signal (arrows) in one study patient. b Section of coronal T2-FLAIR demonstrates the lateral extension of PSD (arrows). Similar sections (from T1-BB) reveal change in signal intensity of PSD from before (Pre) CSF tracer injection (c), and after $3 \mathrm{~h}(\mathbf{d}), 6 \mathrm{~h}(\mathbf{e}), 24 \mathrm{~h}(\mathbf{f})$ and $48 \mathrm{~h}(\mathbf{g}) . \mathrm{ln} \mathbf{~ h}$, the PSD is illustrated in yellow color (for more details, see Supplementary Fig. 1; illustration: Øystein Horgmo, University of Oslo). The percentage change in normalized T1-BB signal units in CSF (blue line) and PSD (red line) for the entire cohort of 18 individuals is presented in $\mathbf{i}$. The signal change was highly significant in both locations (linear mixed model analysis; ${ }^{\star} P<0.05,{ }^{\star \star} P<0.01,{ }^{\star \star \star} P<0.001$ ) and was largest within the CSF. Also note that signal change peaked at $24 \mathrm{~h}$ in both CSF and PSD, and signal increase in these structures was highly associated $(R=0.92$, $P<0.001)$. Error bars refer to $95 \%$ confidence intervals $(\mathrm{Cl})$. 

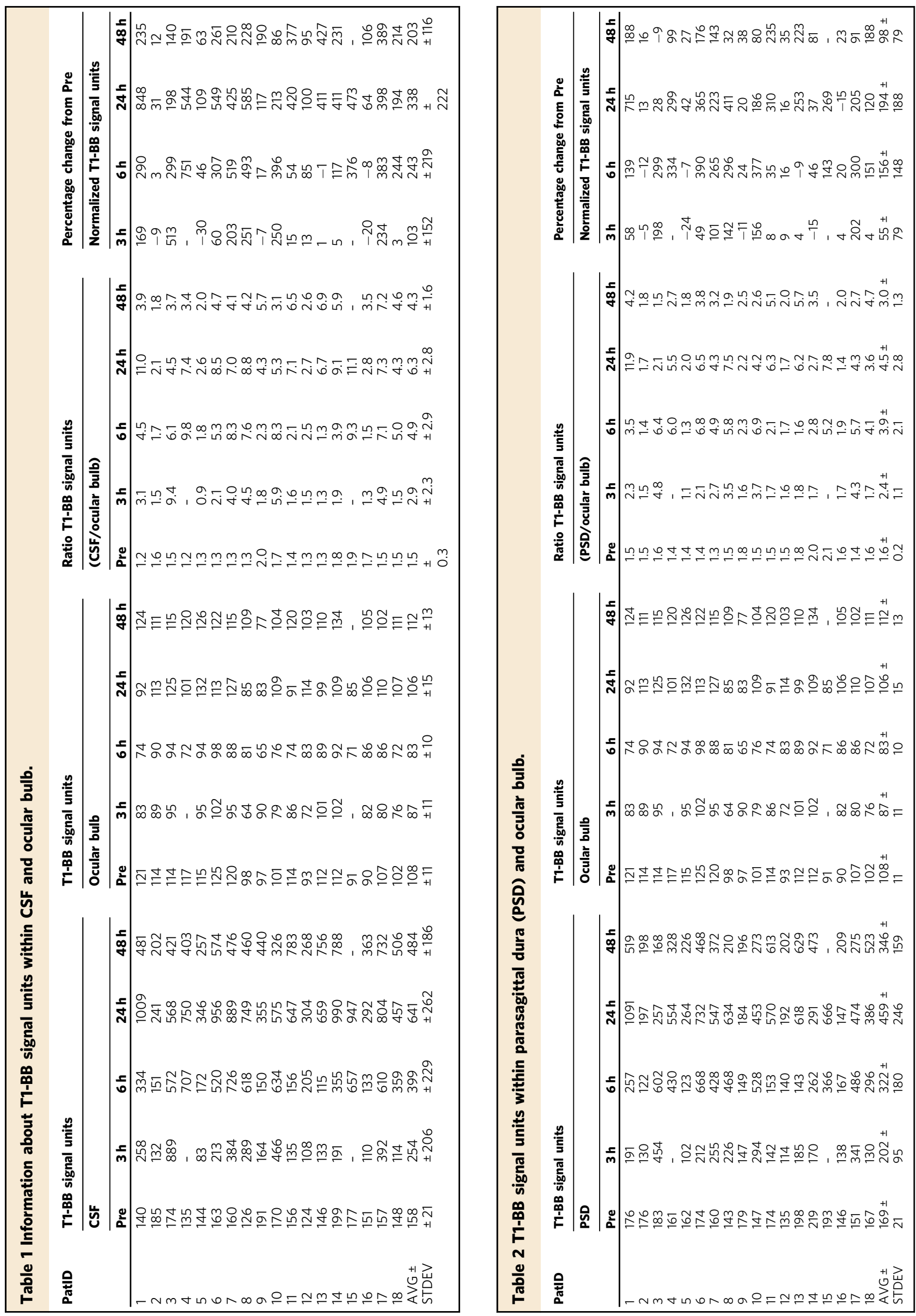

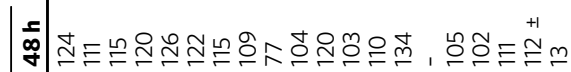

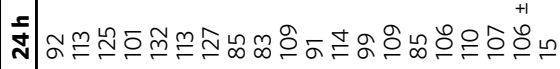

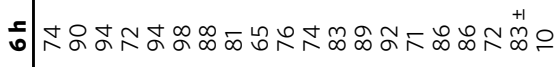

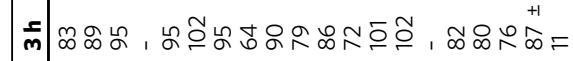

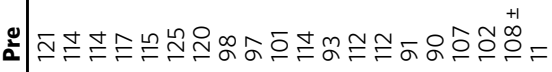

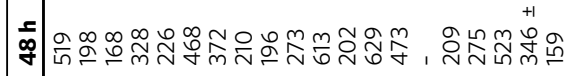

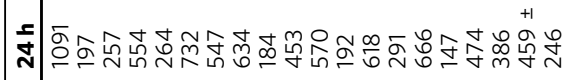

•

m

然

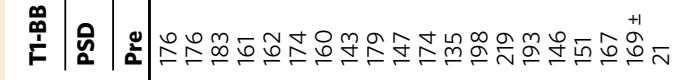



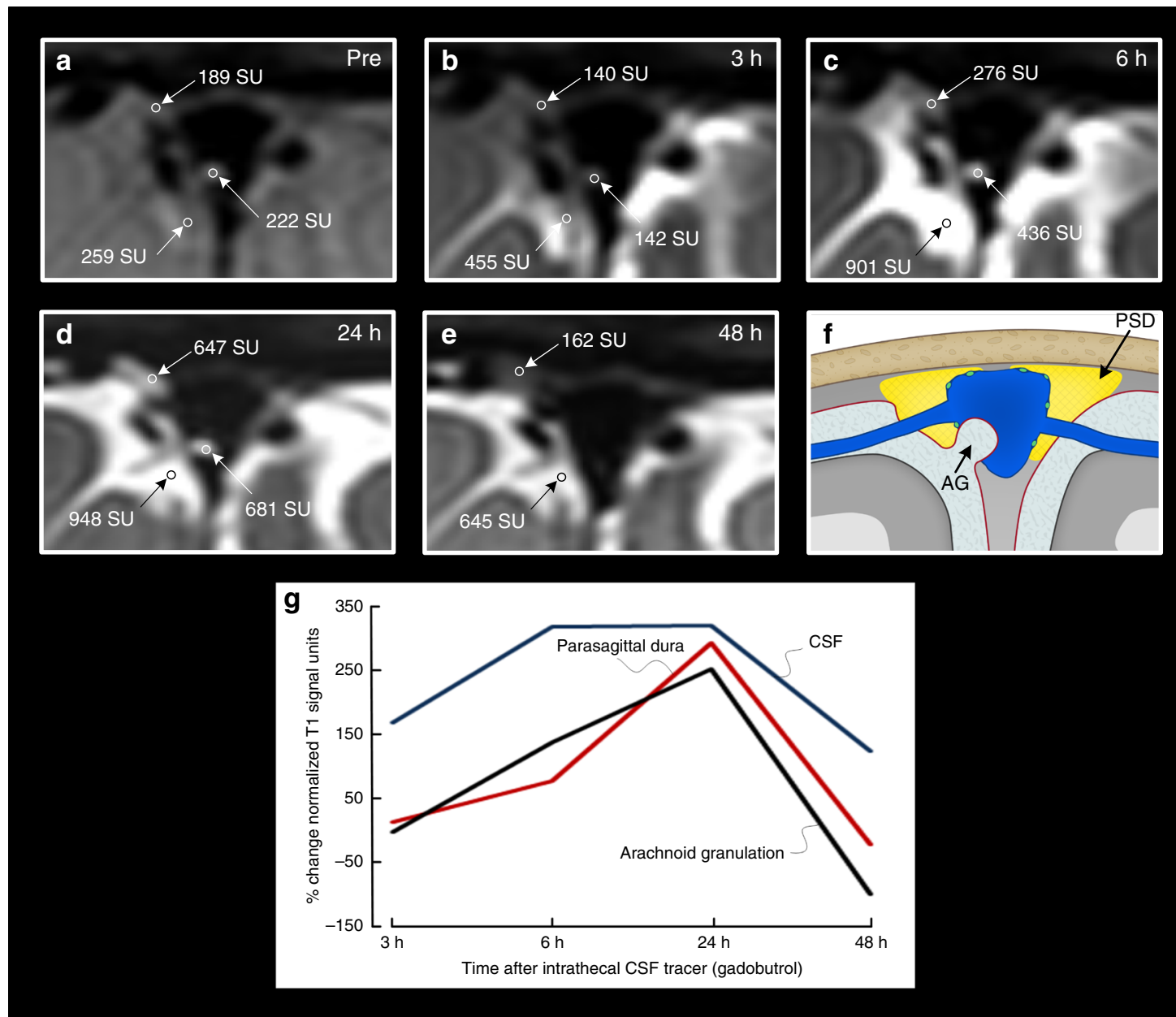

Fig. 3 Visualization of the parasagittal dura and an arachnoid granulation. The T1-BB images are shown for one individual (no. 8) prior to intrathecal gadobutrol injection (a), and after $3 \mathrm{~h}(\mathbf{b}), 6 \mathrm{~h}(\mathbf{c}) 24 \mathrm{~h}$ (d) and $48 \mathrm{~h}$ (e). The enrichment of CSF tracer is quantified as change in signal units (SU) within the parasagittal dura, CSF space and within one arachnoid granulation. The regions of interest are marked by an open circle. At $48 \mathrm{~h}$ (e), enhancement of the arachnoid granulation could no longer be detected. In $\mathbf{f}$ is illustrated the parasagittal dura (PSD, indicated with yellow color) and an arachnoid granulation (AG; illustration: Øystein Horgmo, University of Oslo). In $\mathbf{g}$, the percentage change in normalized T1-BB signal units in CSF (blue line), PSD (red line) and arachnoid granulation (black line) in this individual is presented.

Tracer enrichment in parasagittal dura is furthermore conflicting evidence against a recent animal study, where basal meningeal lymphatic vessels were proposed being more important than dorsal (parasinusoidal) lymphatic vessels for CSF molecular uptake and transport due to their closer proximity to the subarachnoid space in areas with a much thinner dural layer ${ }^{17}$. Even though we also observed signs of lymphatic drainage through basal neuroforamina, the observations do not strengthen the hypothesis that the importance of molecular drainage toward parasagittal structures is inferior to basal outflow. The very thin, linear structures of tracer enhancement we observed below the scull base, and our image resolution with voxel size $1 \mathrm{~mm}$, did not allow us to quantify the relative importance between parasagittal and basal outflow pathways. While our study corroborates findings from animal studies by showing that CSF molecular outflow through skull base foramina occurs also in humans, the presumed importance of the transnasal lymphatic route ${ }^{18}$ requires further exploration in humans. In this study, signs of tracer enhancement below the cribriform plate could be visually confirmed in only $2 / 18$ patients. The olfactory system is indeed of proportionally much lesser size in humans than in rodents, potentially suggesting this molecular clearance route is of less importance, at least relative to clearance toward other cranial nerve outlets at the skull base and the parasagittal dura as corroborated by the current observations.

The present findings also stand in contradistinction to a recent CSF tracer study of mice reporting no signs of tracer propagation through outside the arachnoid layer, and concluding that CSF outflow occurred predominately along cranial nerves through skull neuroforamina ${ }^{7}$. With concern to clearance rate, another rodent study reported very quick exit of tracer from CSF along perineural routes at the skull base ${ }^{19}$. In our human cohort, tracer was present in CSF at a far longer time span (even after 2 days), and the tail of the remaining tracer bolus at late scans along parasagittal dura (Fig. 1; Supplementary Movie 1) may also indicate a net drift of CSF molecules in this direction, rather than toward the skull base. These obvious discrepancies between observations in mice and humans underline the importance of human translational research.

It has previously been shown that neurotoxic molecules such as amyloid- $\beta$ and tau are cleared from brain tissue along paravenous pathways (the glymphatic system) ${ }^{20}$ and speculated that molecules also drain further along cerebral veins along the brain convexities into dural lymphatic vessels ${ }^{4}$. A MRI study suggested the same based on peak CSF tracer enhancement in brain and cervical lymph nodes occurring at the same time point ${ }^{21}$. It has, 


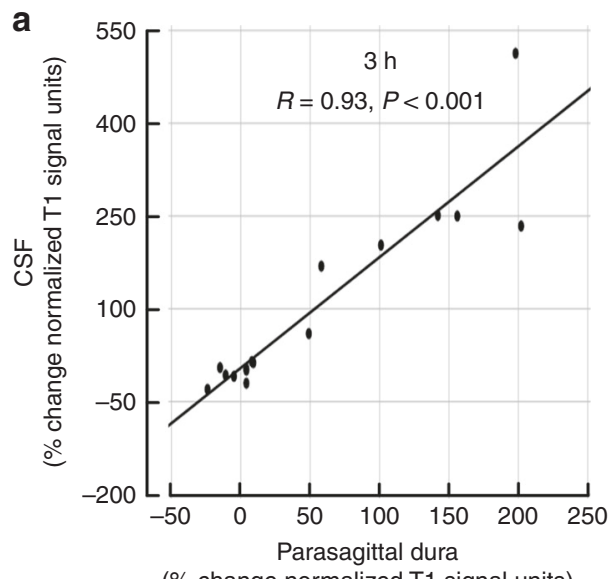

(\% change normalized $\mathrm{T} 1$ signal units)

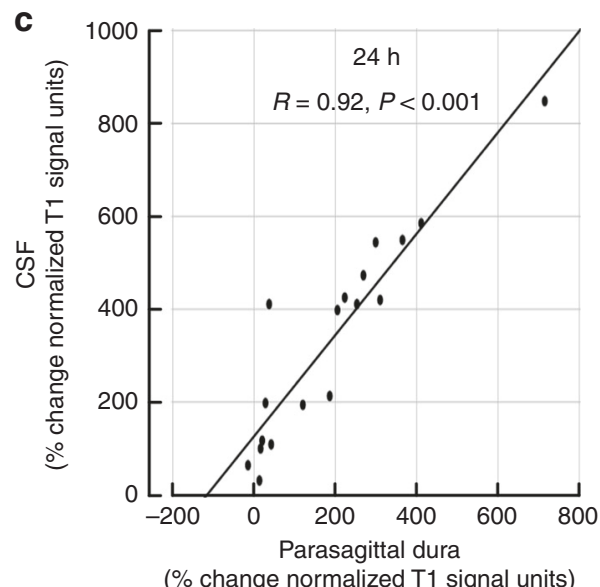

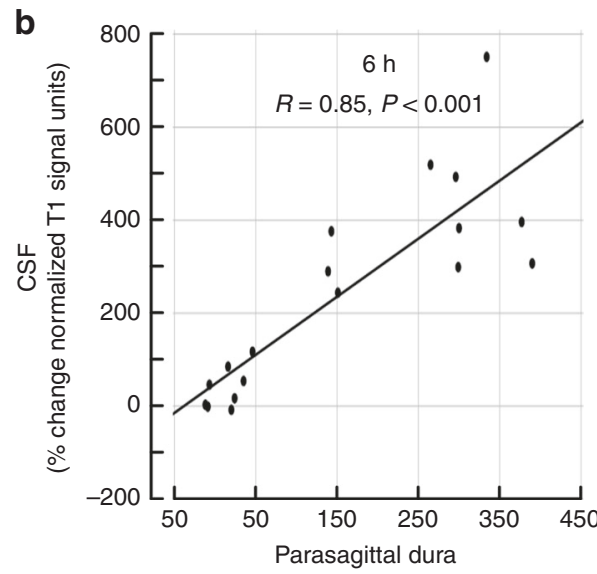

(\% change normalized T1 signal units)

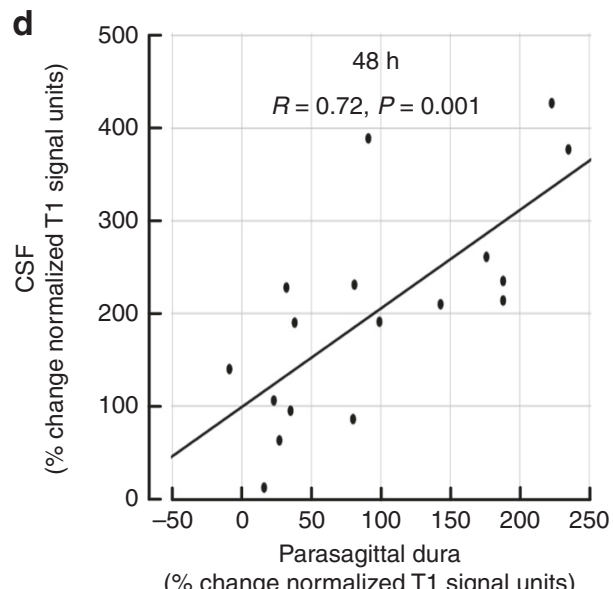

Fig. 4 Correlations between CSF tracer enhancement in parasagittal dura and nearby CSF space. There was a highly significant positive correlation between CSF tracer enhancement within parasagittal dura and nearby CSF spaces at $3 \mathrm{~h} \mathrm{(a),6} \mathrm{h} \mathrm{(b),} 24 \mathrm{~h}$ (c) and $48 \mathrm{~h}$ (d). Each plot presents the fit line and the Pearson correlation coefficient $(R)$ with $P$-value.

however, been demonstrated in rats that from the total amount of MRI contrast agent injected into the cisterna magna, merely $19 \%$ enriched the brain parenchyma, the remainder escaped via other routes ${ }^{22}$. Although the dose, injection site and species differences may be important for this fraction, the present observations of high association between tracer enhancement in CSF and adjacent parasagittal dura suggest that molecules within the CSF primarily drain directly to lymphatic pathways. The extent to which this molecular flux may contribute to brain parenchyma clearance remains to be investigated. In this context, previous findings showing that impairment of meningeal lymphatic function slows paravascular influx of macromolecules into the brain, as well as efflux from the interstitial space ${ }^{5}$, may suggest that impaired lymphatic clearance function may exert its negative effect directly on the accumulation of molecules within the CSF, and thereby further upstream on molecular exit from brain into CSF.

An obvious limitation of this study is the MR image resolution of $1 \mathrm{~mm}$ voxel size. The possible interchange between microscopic contents of the parasagittal dura, such as intradural channels and arachnoid granulations, and dural lymphatic vessels, should be further explored in future studies. In the lack of T1-mapping techniques with image resolution even close to 1 $\mathrm{mm}$, we did not have the opportunity to go beyond semiquantitative measures of tracer enrichment. Ideally, we could also have used an extra-cranial device as reference for normalization of signal units, as the ocular bulbs may be affected by some motion artifacts during scans of several minutes. Moreover, the long duration of molecular clearance from human CSF, and the high demand on patients and resources associated with multiphase MR imaging over $48 \mathrm{~h}$, has limited the number of scans we were able to perform in single patients through this time span. Therefore, the time point for peak tracer enhancement in human parasagittal dura must be considered to represent a rough estimate.

In conclusion, the present study demonstrates in vivo escape of a CSF tracer from the subarachnoid compartment over the arachnoid and into the parasagittal dura along the superior sagittal sinus in humans. The parasagittal dura may thus represent a bridging link for CSF-mediated exchange of molecules between brain tissue and dural lymphatic vessels.

\section{Methods}

Ethical permissions. The study was approved by The Institutional Review Board (2015/1868), Regional Ethics Committee (2015/96) and the National Medicines Agency (15/04932-7). Patients were included after written and oral informed consent. The study was registered in Oslo University Hospital Research Registry (ePhorte 2015/1868)

Experimental design. The study design was prospective and observational: randomization of patients was not relevant; neither was a priori sample size calculation needed. 


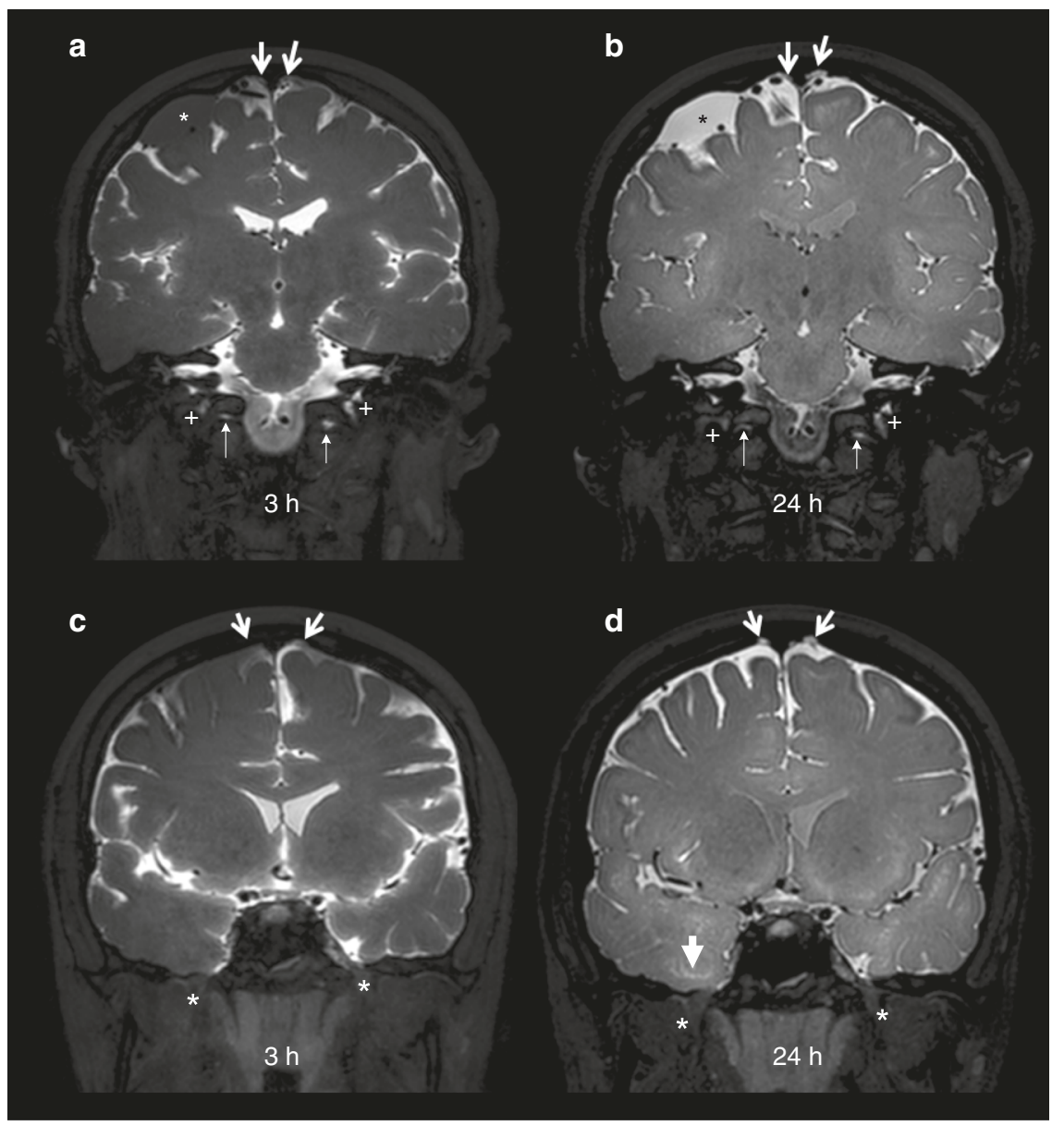

Fig. 5 Evidence for CSF efflux along cranial nerves. Posterior (a, b) and anterior (c, d) mid-coronal T1-BB from a patient (no. 10) under work-up for symptoms possibly related to an arachnoid cyst (asterisks in $\mathbf{a}$ and $\mathbf{b}$, respectively) at the upper convexity of the right cerebral hemisphere. As compared to $3 \mathrm{~h}$ after intrathecal gadobutrol (a), the cyst enhanced homogenously at scans after $24 \mathrm{~h}$ (b). Enrichment of parasagittal dura (thick arrows) increased from 3 $(\mathbf{a}, \mathbf{c})$ to $24 \mathrm{~h}(\mathbf{b}, \mathbf{d})$, respectively. CSF tracer within brain perivascular spaces is well illustrated with T1-BB MRI (exemplified as pointed out by arrowheads; d). In addition is shown CSF tracer enhancement below the skull base outlets of the jugular foramen (crosses) and hypoglossal canal (thin arrows) (a, b). In the same patient, there was no sign of tracer enhancement along the mandibular nerve below level of the oval foramen (asterisks) at any time point (c, d).

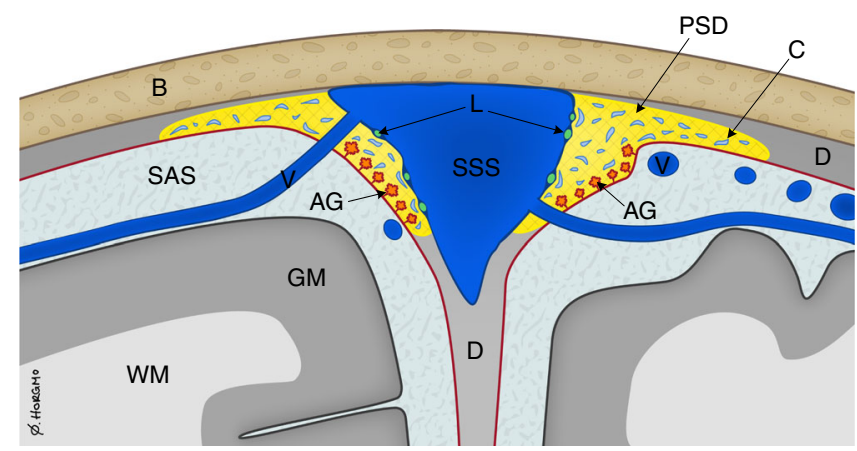

Fig. 6 Cartoon illustrating reported microstructural elements within the parasagittal dura based on refs. ${ }^{\mathbf{1 4}, 15}$. a The parasagittal dura (PSD, colored yellow) contain a network of intradural channels (C, colored light blue), coalescing into lateral lacunae medially (not shown), being in close relation with a dense carpet of intradural arachnoid granulations (AG, orange color). PSD is most prominent at level where the cortical veins ( $V$, dark blue) enter the superior sagittal sinus (SSS, dark blue). Lymphatic vessels ( $L$, green) reside at the SSS wall. The microstructural elements within PSD are of sub-millimeter thickness and therefore not expected to be visualized on MRI with $1 \mathrm{~mm}$ resolution. D dura, GM gray matter, SAS subarachnoid space, WM white matter. Illustrations: Øystein Horgmo, University of Oslo.
Patients. The study included consecutive individuals referred to the Department of neurosurgery, Oslo University Hospital-Rikshospitalet, Oslo, Norway, for various CSF circulation disorders (Supplementary Table 1). Exclusion criteria were: history of hypersensitivity reactions to contrast media agents, history of severe allergy reactions in general, evidence of renal dysfunction (i.e. normal glomerular filtration rate, GFR), age $<18$ or $>80$ years, pregnant or breastfeeding women.

MRI protocol. Scans were obtained in a 3-Tesla MRI unit (Philips Ingenia ${ }^{\circledast}$ ) with a 32-channel head coil.

1. Whole-brain 3D T2-Fluid Attenuated Inversion Recovery (FLAIR), repetition time (TR)/echo time (TE)/inversion time $(\mathrm{TI})=4800 / 311 / 1650$ $\mathrm{ms}$, echo train length $=167$, flip angle $=90^{\circ}, 2$ averages, $1 \times 1 \times 1 \mathrm{~mm}$ voxel size (isotropic), acquisition time $=5 \mathrm{~min}$ and $41 \mathrm{~s}$.

2. Whole-brain 3D T1-Black-blood (BB) scan with $\mathrm{TR} / \mathrm{TE}=700 / 35 \mathrm{~ms}$, echo train length $=55$, flip angle $=80^{\circ}, 2$ averages, $1 \times 1 \times 1 \mathrm{~mm}$ voxel size (isotropic) and acquisition time $4 \mathrm{~min}$ and $54 \mathrm{~s}$.

3. Whole-brain 3D T1-gradient echo (GRE) with $\mathrm{TR}=$ shortest (typically 5.1 $\mathrm{ms}$ ), echo time $=$ shortest (typically $2.3 \mathrm{~ms}$ ), echo train length $=232$, flip angle $=8^{\circ}, 1$ average, $1 \times 1 \times 1 \mathrm{~mm}$ voxel size and acquisition time $6 \mathrm{~min}$ and $29 \mathrm{~s}$.

We repeated the T1-weighted scans at 3,6, 24 and $48 \mathrm{~h}$, respectively, after intrathecal injection of $0.5 \mathrm{ml}$ gadobutrol $(1 \mathrm{mmol} / \mathrm{ml})\left(\right.$ Gadovist $^{\oplus}$, Bayer $\mathrm{AB}$, Sweden). Gadobutrol injection was preceded by injection of $3 \mathrm{ml}$ of $270 \mathrm{mg} \mathrm{I} / \mathrm{ml}$ iodixanol (Visipaque ${ }^{\oplus}$, GE Healthcare, Norway) to ensure correct needle position within the intrathecal compartment. Patients were kept in the supine position with a pillow under their heads until the $6 \mathrm{~h}$ scan was completed and were then allowed to move freely. 
Image analysis. Sagittal T2-fluid FLAIR, as well as T1-BB scans from all time points, were reformatted into coronal and axial 1-mm slices perpendicular and parallel to the plane defined by a line between the anterior and posterior commissures (AC-PC plane), respectively. Identically positioned coronal slices were used to identify parasagittal dura with tissue characteristics remaining unsuppressed (intermediate or high signal) at both T2-FLAIR and T1-BB. T2-FLAIR has the ability to suppress free (unbound) CSF within the ventricular compartment and subarachnoid space ${ }^{23}$. T1-BB images are tuned to darken the contents of blood vessels, even when containing a contrast agent ${ }^{24}$. Thus, in areas with moving protons, such as in arteries and veins, the T1-BB signal is nulled. Around the high brain convexities, there are little motions within the CSF, and therefore the signal is not black (nulled) as in the blood vessels, rendering for a more intermediate CSF-signal.

In areas of parasagittal dura with these features, regions of interest (ROIs) were placed in identical locations at all time points and well within the outer perimeter of parasagittal dura at T1-BB images for robust measurement of $\mathrm{T} 1$ signal change as a sign of tracer enrichment. In addition, a ROI was placed within adjacent subarachnoid space to assess T1 signal change in CSF. The CSF tracer enrichment was measured as change in T1-BB signal units, and was normalized against the reference (i.e. the vitreous body of the ocular bulb) to correct for any baseline shift of image grayscale between time points (Supplementary Fig. 1). We also visually assessed any presence of tracer enhancement on T1-BB images below selected cranial nerve outlets at the skull base and dichotomized this as yes/no whether such enhancement was found present at any time point, or not, respectively.

3D T1-GRE scans were used for segmentation and co-registration rendering for 3D representations (using 3D Slicer version 4 and SPM 12, both open source software) illustrating the relations between the brain surface and CSF tracer enhancement. T1-GRE was, however, unsuitable for precise ROI-placement in parasagittal dura, since the border against the enhancing CSF compartment became effaced at late scans (Supplementary Fig. 2).

Statistics. Statistical analyses were performed using the SPSS software version 22 (IBM Corporation, Armonk, NY). Differences between continuous data were determined using linear mixed models with a random intercept. Correlations were determined by Pearson correlation coefficient. Statistical significance was accepted at the 0.05 level (two-tailed).

Reporting summary. Further information on research design is available in the Nature Research Reporting Summary linked to this article.

\section{Data availability}

The data presented in this work are available upon request.

Received: 17 June 2019; Accepted: 17 December 2019;

Published online: 17 January 2020

\section{References}

1. Ringstad, G. et al. Brain-wide glymphatic enhancement and clearance in humans assessed with MRI. JCI Insight 3, 121537 (2018).

2. Pollay, M. The function and structure of the cerebrospinal fluid outflow system. Cerebrospinal Fluid Res. 7, 9 (2010).

3. Aspelund, A. et al. A dural lymphatic vascular system that drains brain interstitial fluid and macromolecules. J. Exp. Med. 212, 991-999 (2015)

4. Louveau, A. et al. Structural and functional features of central nervous system lymphatic vessels. Nature 523, 337-341 (2015).

5. Da Mesquita, S. et al. Functional aspects of meningeal lymphatics in ageing and Alzheimer's disease. Nature 560, 185-191 (2018).

6. Da Mesquita, S., Fu, Z. \& Kipnis, J. The meningeal lymphatic system: a new player in neurophysiology. Neuron 100, 375-388 (2018).

7. Ma, Q., Ineichen, B. V., Detmar, M. \& Proulx, S. T. Outflow of cerebrospinal fluid is predominantly through lymphatic vessels and is reduced in aged mice. Nat. Commun. 8, 1434 (2017).

8. Nabeshima, S., Reese, T. S., Landis, D. M. \& Brightman, M. W. Junctions in the meninges and marginal glia. J. Comp. Neurol. 164, 127-169 (1975).

9. Coles, J. A., Myburgh, E., Brewer, J. M. \& McMenamin, P. G. Where are we? The anatomy of the murine cortical meninges revisited for intravital imaging, immunology, and clearance of waste from the brain. Prog. Neurobiol. 156, 107-148 (2017).

10. Cheng, K. T. Gadobutrol. in Molecular Imaging and Contrast Agent Database (MICAD) (National Center for Biotechnology Information, Bethesda, USA, 2004). https://www.ncbi.nlm.nih.gov/pubmed/20641787.

11. Absinta, M. et al. Human and nonhuman primate meninges harbor lymphatic vessels that can be visualized noninvasively by MRI. eLife 6, e29738 (2017).
12. Trimble, C. R., Harnsberger, H. R., Castillo, M., Brant-Zawadzki, M. \& Osborn, A. G. "Giant" arachnoid granulations just like CSF?: NOT!! AJNR Am. J. Neuroradiol. 31, 1724-1728 (2010).

13. Mack, J., Squier, W. \& Eastman, J. T. Anatomy and development of the meninges: implications for subdural collections and CSF circulation. Pediatr. Radiol. 39, 200-210 (2009).

14. Han, H., Tao, W. \& Zhang, M. The dural entrance of cerebral bridging vein into the superior sagittal sinus: an anatomical comparison between cadavers and digital subtraction angiography. Neuroradiology 49, 169-175 (2007).

15. Fox, R. J., Walji, A. H., Mielke, B., Petruk, K. C. \& Aronyk, K. E. Anatomic details of intradural channels in the parasagittal dura: a possible pathway for flow of cerebrospinal fluid. Neurosurgery 39, 84-90 (1996).

16. Mastorakos, P. \& McGavern, D. The anatomy and immunology of vasculature in the central nervous system. Sci. Immunol. 4, eaav0492 (2019).

17. Ahn, J. H. et al. Meningeal lymphatic vessels at the skull base drain cerebrospinal fluid. Nature 572, 62-66 (2019)

18. Johnston, M., Zakharov, A., Papaiconomou, C., Salmasi, G. \& Armstrong, D. Evidence of connections between cerebrospinal fluid and nasal lymphatic vessels in humans, non-human primates and other mammalian species. Cerebrospinal Fluid Res. 1, 2 (2004).

19. Ma, Q. et al. Rapid lymphatic efflux limits cerebrospinal fluid flow to the brain. Acta Neuropathol. 137, 151-165 (2019).

20. Iliff, J. J. et al. A paravascular pathway facilitates CSF flow through the brain parenchyma and the clearance of interstitial solutes, including amyloid beta. Sci. Transl. Med. 4, 147ra111 (2012).

21. Eide, P. K., Vatnehol, S. A. S., Emblem, K. E. \& Ringstad, G. Magnetic resonance imaging provides evidence of glymphatic drainage from human brain to cervical lymph nodes. Sci. Rep. 8, 7194 (2018).

22. Lee, H. et al. Quantitative Gd-DOTA uptake from cerebrospinal fluid into rat brain using 3D VFA-SPGR at 9.4T. Magn. Reson. Med. 79, 1568-1578 (2018).

23. Bakshi, R., Ariyaratana, S., Benedict, R. H. \& Jacobs, L. Fluid-attenuated inversion recovery magnetic resonance imaging detects cortical and juxtacortical multiple sclerosis lesions. Arch. Neurol. 58, 742-748 (2001).

24. Mandell, D. M. et al. Intracranial vessel wall MRI: principles and expert consensus recommendations of the american society of neuroradiology. AJNR Am. J. Neuroradiol. 38, 218-229 (2017).

\section{Acknowledgements}

The authors thank Dr. Øivind Gjertsen, Dr. Bård Nedregaard and Dr. Ruth Sletteberg from the Department of Radiology, Oslo University Hospital-Rikshospitalet, who performed the intrathecal gadobutrol injections in all study subjects. We also sincerely thank the Intervention Centre and Department of neurosurgery at Oslo University Hospital Rikshospitalet for providing valuable support with MR scanning and caretaking of all study subjects throughout the study. The author thanks Are Hugo Pripp, $\mathrm{PhD}$, Department of Biostatistics, Epidemiology and Health Economics, Oslo University Hospital, Oslo, for statistical help during preparation of the paper. We also thank Tomas Sakinis, MD at Department of Radiology, Oslo University Hospital, for elaborating 3D representations of imaging data and Øystein Horgmo, University of Oslo, for providing illustrations.

\section{Author contributions}

Conceptualization and design, G.R. and P.K.E; investigation, formal analysis and visualization, G.R. and P.K.E.; supervision, administration and writing-original draft, G. R. and P.K.E.; writing-review and editing, G.R. and P.K.E. Both authors approved the final manuscript.

\section{Competing interests}

Geir Ringstad receives a fee for speaking at the Bayer symposium at the European Congress of Radiology 2020 (Vienna, Austria). The authors declare no other competing interests.

\section{Additional information}

Supplementary information is available for this paper at https://doi.org/10.1038/s41467 019-14195-x.

Correspondence and requests for materials should be addressed to P.K.E.

Peer review information Nature Communications thanks Helene Benveniste, Govind Nair and the other, anonymous, reviewer(s) for their contribution to the peer review of this work. Peer reviewer reports are available.

Reprints and permission information is available at http://www.nature.com/reprints Publisher's note Springer Nature remains neutral with regard to jurisdictional claims in published maps and institutional affiliations. 
(c) (i) Open Access This article is licensed under a Creative Commons Attribution 4.0 International License, which permits use, sharing, adaptation, distribution and reproduction in any medium or format, as long as you give appropriate credit to the original author(s) and the source, provide a link to the Creative Commons license, and indicate if changes were made. The images or other third party material in this article are included in the article's Creative Commons license, unless indicated otherwise in a credit line to the material. If material is not included in the article's Creative Commons license and your intended use is not permitted by statutory regulation or exceeds the permitted use, you will need to obtain permission directly from the copyright holder. To view a copy of this license, visit http://creativecommons.org/ licenses/by/4.0/.

(C) The Author(s) 2020 